\title{
Um Caso de Mobilidade Dentária e Tumefação Maxilar
}

\section{A Case of Tooth Looseness and Maxillary Tumefaction}

\author{
Daniela Abreu Silva1*, Francisco Macedo², Dolores Quintal ${ }^{3}$
}

PALAVRAS-CHAVE: Doenças Maxilares; Quisto Radicular

KEYWORDS: Maxillary Diseases; Radicular Cyst

Os quistos radiculares são os quistos odontogénicos mais comuns. São formados pela proliferação celular dos restos epiteliais de Malassez, secundária à inflamação prolongada da polpa dentária. ${ }^{1-4}$ Estão habitualmente associados a dentes com lesões cariosas ou fraturas. ${ }^{1-3}$ Ocorrem mais frequentemente no sexo masculino, e na terceira e sexta décadas de vida. ${ }^{3}$ Geralmente apresentam dimensões entre 0,5 e 1,5cm e são assintomáticos. Contudo, podem atingir maiores dimensões, com os seguintes sinais e sintomas: odontalgias, tumefação, mobilidade dentária, desvio de estruturas e destruição óssea. 2,3 Apesar de os quistos odontogénicos serem lesões benignas, podem sofrer transformação maligna em 0,13\%-3\%, devido à inflamação crónica. ${ }^{3,5}$
Apresentamos um caso clínico de uma mulher de 43 anos, com antecedentes de diabetes, neoplasia mamária e ex-fumadora, com quadro de vários episódios de odontalgias e mobilidade dentária na arcada dentária superior, e ainda tumefação crescente na região pré-maxilar, com 8 meses de evolução. Realizou tomografia computorizada dos seios perinasais (Fig. 1) e ortopantomografia (Fig. 2), que mostraram volumosa imagem lacunar óssea, adjacente às raízes dentárias, na região anterior paramediana da arcada alveolar superior esquerda, compatível com quisto radicular do maxilar, que condicionava desvio do septo nasal. Perante a hipótese diagnóstica colocada, foi realizada apicetomia dos dentes 11, 12, 21 , 22 e 23, enucleação e curetagem do quisto, que tinha

1. Médica Interna da Formação Específica em Medicina Geral e Familiar no ACES Madeira - Centro de Saúde do Bom Jesus, Funchal, Portugal. 2. Médico Assistente de Medicina Geral e Familiar no ACES Madeira - Centro de Saúde do Bom Jesus, Funchal, Portugal. 3. Médica Assistente Graduada Sénior de Medicina Geral e Familiar no ACES Madeira - Centro de Saúde do Caniço, Santa Cruz, e Coordenadora do Internato Médico de Medicina Geral e Familiar na Região Autónoma da Madeira, Portugal. Recebido/Received: 09/11/2020 - Aceite/Accepted: 24/03/2021 - Publicado online/Published online: 16/04/2021 - Publicado/Published: 30/06/2021

• Autor (es) (ou seu (s) empregador (es)) e Gazeta Médica 2021. Reutilização permitida de acordo com CC BY-NC. Nenhuma reutilização comercial. ${ }^{\bullet}$ Author(s) (or their employer(s)) and Gazeta Médica 2021. Re-use permitted under CC BY-NC. No commercial re-use. 


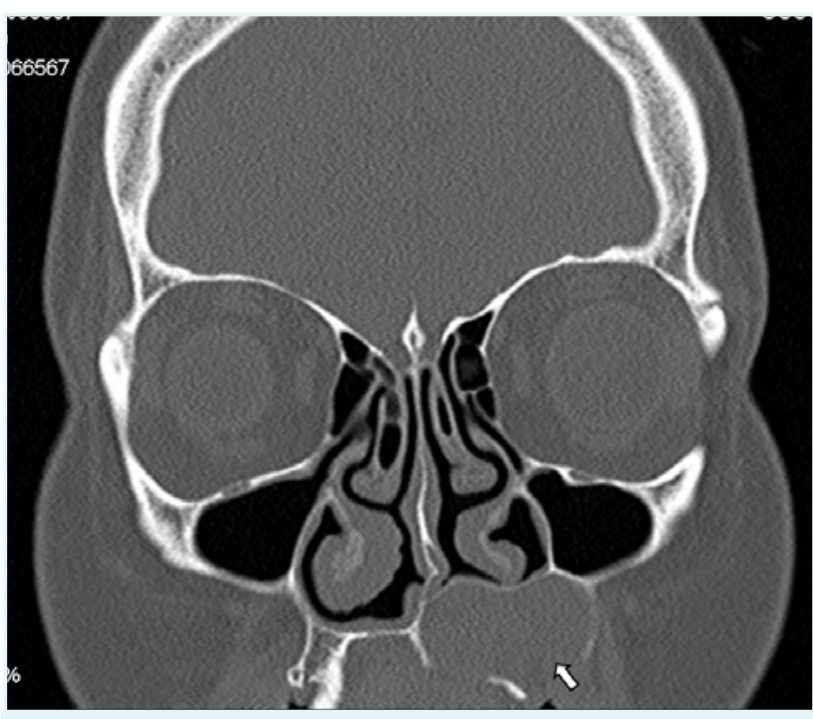

FIGURA 1. Tomografia computorizada mostrando formação quística maxilar (assinalado pela seta) com 4,6 cm de maior diâmetro.

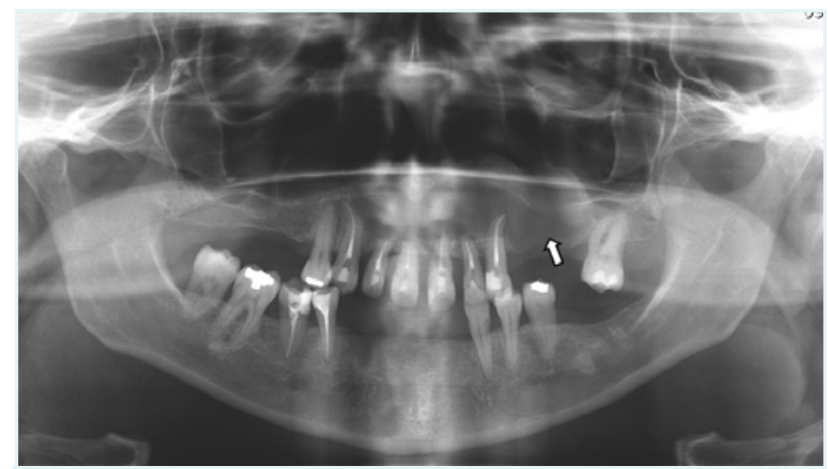

FIGURA 2. Ortopantomografia mostrando formação quística maxilar (assinalado pela seta).

4,3×2,3 cm de dimensão. O exame anátomo-patológico confirmou o diagnóstico de quisto radicular do maxilar.

A maioria dos quistos radiculares podem ser tratados conservadoramente, com terapêutica endodôntica e eventual descompressão do quisto. Quando atingem grandes dimensões, deve proceder-se à sua enucleação e eventual extração dos dentes envolvidos. ${ }^{1-3,6}$

Este caso clínico ilustra a importância de aumentar o índice de suspeita clínica para os quistos radiculares, perante odontalgia de repetição e mobilidade dentária, permitindo o diagnóstico e tratamento precoces, de forma a evitar complicações como mobilidade e perda de peças dentárias, desvio de estruturas, destruição óssea e malignização.

\section{RESPONSABILIDADES ÉTICAS}

CONFLITOS DE INTERESSE: Os autores declaram a inexistência de conflitos de interesse na realização do presente trabalho.

FONTES DE FINANCIAMENTO: Não existiram fontes externas de financiamento para a realização deste artigo.
CONFIDENCIALIDADE DOS DADOS: Os autores declaram ter seguido os protocolos da sua instituição acerca da publicação dos dados de doentes.

CONSENTIMENTO: Consentimento do doente para publicação obtido.

PROVENIÊNCIA E REVISÃO POR PARES: Não comissionado; revisão externa por pares.

\section{ETHICAL DISCLOSURES}

CONFLICTS OF INTEREST: The authors have no conflicts of interest to declare.

FINANCING SUPPORT: This work has not received any contribution, grant or scholarship.

CONFIDENTIALITY OF DATA: The authors declare that they have followed the protocols of their work center on the publication of data from patients.

PATIENT CONSENT: Consent for publication was obtained.

PROVENANCE AND PEER REVIEW: Not commissioned; externally peer reviewed.

\section{REFERÊNCIAS}

1. Li Bernardi L, Visioli F, Nör C, Rados PV. Radicular Cyst: An Update of the Biological Factors Related to Lining Epithelium. J Endod. 2015;41:1951-61. doi:10.1016/j.joen.2015.08.036.

2. Hahn HM, Lee YJ, Park DH. Huge radicular cyst of the maxilla treated with complete resection and immediate reconstruction by rib bone graft. J Maxillofac Oral Surg. 2018;18:378-81. doi: 10.1007/s12663-018-1125-0.

3. Deshmukh J, Shrivastava R, Bharath KP, Mallikarjuna R. Giant radicular cyst of the maxilla. BMJ Case Rep. 2014; 2014: bcr2014203678. doi: 10.1136/bcr-2014- 203678.

4. Johnson NR, Gannon OM, Savage NW, Batstone MD. Frequency of odontogenic cysts and tumors: a systematic review. J Investig Clin Dent. 2013;4:1-7. doi: 10.1111/jicd.12044.

5. Borrás-Ferreres J, Sánchez-Torres A, Gay-Escoda C. Malignant changes developing from odontogenic cysts: A systematic review. J Clin Exp Dent. 2016;8:e622-8. doi: 10.4317/ jced.53256.

6. Matijević S, Jovičić B, Bubalo M, Dukić S, Čutović T. Treatment of a large radicular cyst - enucleation or decompression? Vojnosanit Pregl. 2015;72:372-4. doi: 10.2298/NSP1504372M. 